\title{
Violencia intrafamiliar y factores de riesgo en mujeres afrodescendientes de la ciudad de Cartagena
}

\author{
Shyrley Díaz Cárdenas ${ }^{\mathrm{a}}$, Katherine Margarita Arrieta Vergara ${ }^{\mathrm{b}}$, Farith González Martínezc.
}

\author{
a Odontóloga, Magister en \\ Salud Pública, especialista en \\ Salud Familiar y Gerencia en \\ Salud. \\ b Odontóloga, Magíster en \\ Salud Pública, especialista en \\ Gerencia en Salud. \\ c Odontólogo, Magister en \\ Salud Pública, especialista en \\ Investigación Social. \\ Docentes de la Facultad de \\ Odontología de la \\ Universidad de Cartagena \\ (Colombia)
}

Correspondencia: Shyrley Díaz Cárdenas. Facultad de Odontología. Universidad de Cartagena (Colombia) Correo electrónico: shyrley77@hotmail.com

Recibido el 10 de octubre de 2014.

Aceptado para su publicación el 19 de enero de 2015 .

\section{RESUMEN}

Objetivo: Estimar la asociación entre violencia intrafamiliar y factores de riesgo en mujeres afrodescendientes de la ciudad de Cartagena.

Diseño del estudio: Estudio transversal.

Emplazamiento: Comunidad Rural de Tierra Baja, Cartagena de Indias, Colombia.

Participantes: 220 mujeres, seleccionadas a conveniencia.

Métodos: Se indagó por factores sociodemográficos, presencia de violencia y abuso, y su severidad (cuestionario de abuso Norvold [NorAQ]), causas de actos violentos durante el último mes, factores de riesgo relacionados (antecedentes de violencia, roles, consumo de alcohol, falta de recreación, funcionalidad familiar [Apgar Familiar]). Los datos fueron analizados a partir de proporciones y la asociación se obtuvo por razones de disparidad y regresión logística nominal.

Resultados: El 20,4\% (IC95\% 15,1-25,7) de las mujeres manifiestan ser víctimas de violencia, en su mayoría de abuso emocional, siendo el esposo el principal agresor; el $66,4 \%$ no denunciaron los casos por miedo y los celos son la principal causa que la desencadenó. En el análisis multivariado, el modelo que mejor explica la presencia de violencia hacia la mujer corresponde a los siguientes factores: estado civil, falta de recreación y consumo de alcohol $(p=0,0001)$.

Conclusiones: Las mujeres de Tierra Baja sufren de violencia, posiblemente por falta de oportunidades de recreación y consumo de alcohol de su pareja, lo que no garantiza el bienestar biopsicosocial de sus familias.

PALABRAS CLAVE: Mujeres. Violencia familiar. Riesgo. Medicina familiar y comunitaria.

\section{ABSTRACT}

Domestic violence and risk factors in women of African descent of the city of Cartagena

Objective: To estimate the association between family violence and risk factors in afro descendent women from the city of Cartagena.

Study design: Cross-Sectional Study Cross-Sectional Study

Location: Tierra Baja Rural Community, Cartagena de Indias, Colombia.

Participants: 220 women selected by convenience sample.

Methods: The factors investigated included sociodemographic factors, presence of violence and abuse and its severity (Norvold abuse questionnaire [NorAQ]), causes of violence during the last month, related risk factors (history of violence, roles, alcohol consumption, lack of recreation, family functioning [Family Apgar]). Data were described using proportions, and the association was obtained with odds ratios by nominal logistic regression.

Results: $20.4 \%$ (95\% Cl 15.1-25.7) of women report being victims of violence, mostly emotional abuse, being the husband the primary aggressor; $66,4 \%$ did not report cases for fear, and jealousy is the main trigger. In the multivariate analysis, the model that best explains the presence of violence against women corresponds to the following factors: marital status, lack of recreation and alcohol consumption $(p=0.0001)$.

Conclusions: Women from Tierra Baja suffer violence, possibly due to lack of opportunities for recreation and to their partner's alcohol abuse, which does not guarantee the biopsychosocial welfare of their families.

KEY WORDS: Women. Family violence. Risk. Family and community medicine. 


\section{INTRODUCCIÓN}

La violencia contra la mujer es un problema de salud pública en nuestra sociedad ${ }^{1}$; ha estado presente desde los primeros vestigios en la historia evolutiva del hombre, penetrando en las diferentes formaciones económicas y sociales..$^{2,3}$ Se observa en todas las sociedades del mundo, sin distinción de posición económica, raza o cultura, habiéndose llegado a considerar como parte de una cultura y, en cierto modo, "aceptándose" como un integrante más en algunos núcleos familiares. Por lo tanto, la violencia machista puede ser considerada un problema mundial, histórico y estructural. ${ }^{4}$

Las Naciones Unidas definen la violencia contra la mujer como "todo acto de violencia basado en el género que tiene como resultado posible o real un daño físico, sexual o psicológico, incluidas las amenazas, la coerción o la privación arbitraria de la libertad, ya sea que ocurra en la vida pública o en la vida privada". ${ }^{1}$ El $35 \%$ de las mujeres del mundo han sido víctimas de violencia física y/o sexual por parte de su pareja o de personas distintas a ésta, siendo este un elevado porcentaje de la población femenina mundial. ${ }^{5}$ En Colombia, se conjuga un clima de violencia y discriminación contra la mujer, bajo el contexto de conflicto armado que la acentúa; el Instituto Nacional de Medicina Legal y Ciencias Forenses revela en los seis primeros meses del año 2013, 514 mujeres víctimas de homicidio, reportándose como principal causa la violencia de pareja, con 12048 mujeres víctimas, seguido de los delitos sexuales. ${ }^{6}$ En Cartagena, según el Centro del Observatorio y del Delito COSED, en el primer trimestre del año 2013 se denunciaron 458 casos de violencia, de los cuales el maltrato a la pareja representa más del $63 \%$ del total de casos denunciados. $^{7}$

La violencia hacia la mujer es un suceso complejo, multifactorial; uno de ellos es la permanencia de unas estructuras jerárquicas patriarcales que reproducen una cultura donde las mujeres son vistas como personas "maltratables" y no tenidas en cuenta para la toma de decisiones. ${ }^{4}$ Prueba de ello es que las reiterativas violaciones a los derechos de las mujeres se desarrollan tanto en tiempos de paz como en tiempos de conflicto armado, atendiendo a diversos contextos y a imaginarios culturales similares, que limitan y atentan contra la libertad femenina y el desarrollo de sus capacidades. ${ }^{4}$ Muchas mujeres se involucran en este fenómeno por diversos factores, como la baja escolaridad, lo que genera menos oportunidades laborales y dependencia económica de sus esposos, disfun- cionalidad familiar, baja autoestima, experiencia previa de violencia en la familia de origen y en la del compañero, uso de alcohol y drogas, rigidez y sobrecarga de roles en la familia, entre otros. ${ }^{8}$

También el factor cultural influye en la aparición de la violencia. ${ }^{9}$ En la familia se reproducen relaciones de poder donde se desarrollan sutiles formas de violencia. ${ }^{10}$ De acuerdo al funcionamiento del poder, en la esfera privada existen jerarquías asumidas socialmente como "naturales", que se legitiman de manera permanente mediante el monopolio de la violencia como principal aliado. ${ }^{11}$ Además, se destaca que "dentro de la pirámide familiar, las víctimas del ejercicio del poder a través de medios violentos son principalmente las mujeres, las niñas y los niños". ${ }^{11}$ En los países de América Latina aún sigue siendo común que se fundan las relaciones intrafamiliares en el sometimiento de las mujeres y los niños al poder masculino. ${ }^{12}$

Las consecuencias de la violencia contra las mujeres son muy amplias e influyen en todos los aspectos de su vida, su salud y la de sus hijos y se extienden, además, al conjunto de la sociedad. ${ }^{13}$ El impacto de la violencia sobre la salud mental de las mujeres presenta consecuencias devastadoras, como elevada incidencia de tensión nerviosa, ataques de pánico, trastornos del sueño, alcoholismo, abuso de drogas, baja autoestima, trastorno por estrés postraumático y depresión. ${ }^{14}$ Esta situación se convierte en un factor de riesgo para su propia salud, la de sus hijos y su familia, impidiendo el buen funcionamiento familiar y el crecimiento de cada uno de sus integrantes.

A pesar de ser la violencia hacia la mujer un hecho tan común y constatado, son pocos los estudios a nivel local y nacional que investigan este problema, por eso el objetivo de este estudio es estimar la asociación entre la violencia intrafamiliar y factores de riesgo en mujeres afro descendientes de Tierra Baja, de la Ciudad de Cartagena, beneficiarias de un programa de promoción y prevención en salud bucal de la Facultad de Odontología de la Universidad de Cartagena, que integralmente busca proteger los núcleos familiares de los niños, favorecidos también de estos programas ofertados a la comunidad, y principales víctimas de este proceso social.

\section{MATERIAL Y MÉTODOS}

Estudio de corte transversal realizado en una población de referencia de 300 mujeres de la vereda de Tierra Baja, del corregimiento Boquilla, de 
la ciudad de Cartagena de Indias, Colombia, en el segundo semestre 2013. Se trata de beneficiarias de las prácticas comunitarias de salud bucal de la Facultad de Odontología de la Universidad de Cartagena. Se incluyeron todas las mujeres nacidas en la comunidad (afro descendientes) con relación conyugal vigente o pasada y que aceptaran participar en el estudio, teniendo en cuenta las disposiciones éticas soportados por la normativa internacional (Declaración de Helsinki y modificación de Edimburgo 2000) y la nacional para Colombia (Resolución 8430, de 1993, del Ministerio de la Salud). Se excluyeron a mujeres con discapacidad física o mental para cumplimentar el cuestionario. En el presente estudio participaron 220 mujeres en total.

Se aplicó un cuestionario de auto-informe, anónimo, que contenía 76 preguntas de selección dicotómicas y politómicas, con única respuesta, diseñado desde la teoría y evaluada su validez de apariencia por tres jueces expertos, teniendo en cuenta su pertinencia, suficiencia de las categorías, plausibilidad, semántica, sintaxis y ordenamiento de los ítems. La validación del instrumento se puso a prueba en un pilotaje, con un grupo de mujeres con características similares y la persona que entregó los cuestionarios (una mujer facilitadora de la participación) fue entrenada, quien garantizó el anonimato y confidencialidad de la información a través del uso de una urna sellada. El cuestionario contenía la siguiente información: características socio-demográficas (edad, escolaridad, estado civil, ocupación, ingresos socioeconómicos), percepción de sufrir violencia y tipos de abusos experimentados en el último mes. Se utilizó para esto último el Cuestionario de Abuso Norvold (NorAQ), el cual fue diseñado en Suiza por Katarina ${ }^{15}$, y dada su buena fiabilidad test-retest con valores desde 0,81 y estandarización en cuanto a la evaluación de los diferentes tipos de abuso, se realizó en este estudio la adaptación cultural evaluada dentro del contexto, a través de dos pruebas piloto, midiéndose la confiabilidad-estabilidad test-retest en 100 mujeres con características similares a la del estudio, con resultados de 0,73. Este instrumento consta de 13 preguntas divididas en cuatro tipos de maltrato: emocional o psicológico (gritos, ofensas, humillaciones, amenazas), físico (lanzar objetos, empujones, sacudidas, bofetadas), sexual y abuso en los servicios de salud..$^{15}$ El contenido de las preguntas varía desde abuso leve a grave, lo que permite una clasificación aproximada de la gravedad de cualquier acto abusivo. Las mujeres que reportaron sufrir más de un tipo específico de abuso eran clasificadas de acuerdo con el acto abusivo más grave. El abuso emocional, físico y sexual, y el abuso en la sistema de atención de la salud se define como haber contestado sí a uno o varios de las tres / cuatro preguntas sobre cada tipo de abuso en NorAQ. Si una mujer había sufrido abuso, seguía respondiendo a las preguntas más detalladas; por ejemplo, quién fue el autor del abuso, cuando ocurrió el abuso y si alguna vez le había dicho a alguien sobre lo que pasó. Para evaluar el "sufrimiento actual "se midió una escala de 11 puntos $(0=\sin$ dolor, $10=$ sufre terriblemente). El cuestionario se cierra con preguntas generales sobre abuso, como nunca haber denunciado a la policía o el temor a ser víctima de abuso en el futuro.

También se indagó por conocimientos, actitudes, prácticas y mitos en torno al manejo de la violencia contra la mujer, y factores de riesgo, como antecedentes de violencia, roles, consumo de alcohol, dependencia económica, problemas conyugales, comunicación con la pareja, funcionalidad familiar y soporte de amigos; estos dos últimos evaluados a través del Apgar Familiar. ${ }^{16}$ Este último consta de cinco preguntas tipo Lickert que se puntúan de 0 a 4 ("siempre" 4 puntos, "casi siempre" 3 puntos, "algunas veces" 2 puntos, "casi nunca" 1 punto, "nunca" 0), los resultados pueden variar entre 0 y 20 puntos, puntuaciones entre 18 y 20 sugieren funcionalidad familiar, e inferiores a 18 , disfuncionalidad familiar. Esta escala, traducida y validada en castellano, tiene validez de constructo y la validez factorial, presenta una consistencia interna de 0,81 y la confiabilidad prueba-reprueba de $0,81 .{ }^{16}$

El análisis de la información se realizó con el programa para Windows STATA $12.0{ }^{\circledR}$. Los datos fueron analizados a partir de estadística descriptiva (medias, desviaciones estándar, frecuencias absolutas y relativas). Se estimaron por proporciones la ocurrencia de violencia hacia la mujer, así como el abuso y severidad del abuso. Como variables explicatorias se determinaron los conocimientos, actitudes y prácticas y otros factores considerados como de riesgo. Para las asociaciones, se utilizaron razones de disparidad (Odds Ratio), con estimadores ajustados por análisis múltiple e intervalos de confianza del 95\%. A nivel mutivariable se utilizó regresión logística nominal, con inclusión en los modelos de los factores que mostraron probabilidades inferiores a 0,20; se conformaron modelos a partir de la exclusión de cada una de las variables en un proceso de pasos hacia atrás según las recomendaciones de Greenland. ${ }^{17}$ Además, fue usada la bondad del ajuste de Hosmer-Lemeshow ${ }^{18}$, con el fin de evidenciar la significancia del mejor modelo a partir de valores de $p>0,10$. 


\section{RESULTADOS}

De las 220 mujeres afro descendientes participantes, la gran mayoría se encontraban en etapa de adultez joven (20 a 44 años), con una media de 36 años $(\mathrm{DE}=8)$; presentaban bajo nivel de escolaridad y más de la mitad vivían en unión libre con su pareja. Eran amas de casa cuyas familias cuentan con ingresos mensuales menores a un Salario Mínimo Mensual Legal Vigente (SMMLV) para satisfacer necesidades básicas (menos de 585.000 pesos colombianos) y el 46,8\% llevan más de 10 años conviviendo con su pareja (tabla 1).

Cuarenta y cinco mujeres $(20,4 \%$, IC95\%: $15,1-$ $25,7)$ refieren estar sufriendo violencia durante el

\begin{tabular}{|c|c|c|}
\hline Variables & $\begin{array}{c}\text { Frecuencia } \\
(\mathrm{N}=220)\end{array}$ & Porcentaje \\
\hline \multicolumn{3}{|l|}{ Edad } \\
\hline Menor de 19 años & 8 & 3,6 \\
\hline 19-44 años & 151 & 68,6 \\
\hline 45-59 años & 49 & 22,3 \\
\hline Mayores de 60 años & 12 & 5,4 \\
\hline \multicolumn{3}{|l|}{ Escolaridad } \\
\hline Analfabeta & 3 & 1,4 \\
\hline Primaria Incompleta & 51 & 23,2 \\
\hline Primaria Completa & 33 & 15 \\
\hline Secundaria Incompleta & 70 & 31,8 \\
\hline Secundaria Completa & 39 & 17,7 \\
\hline Técnico o Tecnólogo & 19 & 8,6 \\
\hline Universitario & 5 & 2,3 \\
\hline \multicolumn{3}{|l|}{ Estado civil } \\
\hline Soltera & 13 & 5,9 \\
\hline Casada & 44 & 20 \\
\hline Unión Libre & 145 & 65,9 \\
\hline Separada & 11 & 5 \\
\hline Divorciada & 3 & 1,4 \\
\hline Viuda & 4 & 1,8 \\
\hline \multicolumn{3}{|l|}{ Ocupación } \\
\hline Amas de Casa & 166 & 75,4 \\
\hline Profesionales Universitarios & 5 & 2,3 \\
\hline Trabajadores no Calificados & 46 & 20,9 \\
\hline Técnicos, postsecundarios no universitarios & 1 & 0,4 \\
\hline Empleados de Oficina & 2 & 0,9 \\
\hline \multicolumn{3}{|l|}{ Ingresos económicos de la familia } \\
\hline Menos de 1 SMMLV & 154 & 70 \\
\hline Entre 1 y 2 SMMLV & 60 & 27,2 \\
\hline Más de 2 SMMLV & 6 & 2,7 \\
\hline
\end{tabular}

Tabla 1. Características socio-demográficas en las mujeres de la comunidad de Tierra Baja - Boquilla, Cartagena. 
último mes. El tipo de abuso que más se presentó, según el cuestionario NorAQ, fue el abuso emocional (tabla 2). Con respecto al abuso físico, la zona del cuerpo más afectada fue la cara $(8,2 \%$; IC95\%: 4,5-11) y la principal persona agresora corresponde al esposo (75\% IC95\%: 60-89); entre el 4 y 18 $\%$ de las mujeres alguna vez se les ha prohibido trabajar, estudiar y se les han controlado actos como llamadas telefónicas, visitas a las amigas o vecinos $(2,7 \%$; IC95\%: 0,5-4,8)

$\mathrm{Al}$ indagar por la presencia de factores de riesgo de violencia (tabla 3), se hallaron que los esposos presentaban antecedentes de depresión y enfermedades mentales, antecedentes de violencia en

\begin{tabular}{l} 
Preguntas para evaluar abuso según el NorAQ Abuse \\
\hline Abuso Emocional \\
¿De forma continua y por un tiempo prolongado la han reprimi- \\
do, degradado o humillado? \\
¿De forma continua y bajo amenaza y fuerza le han controlado el \\
contacto con otras personas, o le han controlado lo que puede o \\
no hacer?
\end{tabular}

¿Ha vivido con miedo porque alguien la ha amenazado o a alguien cercano a usted?

\begin{tabular}{cccc} 
& Si & \multicolumn{3}{c}{ No } \\
\hline $\mathbf{n}$ & $\%$ & $\mathbf{n}$ & $\%$ \\
56 & 25,4 & 164 & 74,6 \\
37 & 16,8 & 183 & 83,2 \\
& & & \\
25 & 11,4 & 195 & 88,6
\end{tabular}

\section{Abuso Físico}

¿Alguna vez alguien la ha sujetado con firmeza en contra de su voluntad o le han golpeado su cara?

¿Le han dado palizas, golpes, golpeado con algún objeto duro, pateado o empujado violentamente o le han hecho algo similar?

¿Alguien ha atentado o amenazado contra su vida con un arma o cuchillo o estrangulándole o con cualquier otro acto similar?

\section{Abuso Sexual}

¿Alguien le ha tocado partes de su cuerpo o la han obligado a tocar partes de su propio cuerpo diferentes a los genitales de una "forma sexual"?

¿La han humillado sexualmente, por ejemplo obligándola a ver o participar en una película porno o similar, o a mostrar su cuerpo desnudo?

¿En contra de su voluntad alguien ha tocado sus genitales o utiliza su cuerpo para satisfacerse sexualmente o la obliga a tocar los genitales de otra persona?

¿Alguien en contra de su voluntad ha introducido su pene u otro objeto en la vagina, boca o recto?

\section{Abuso en los Servicios de la Salud}

¿Se ha sentido ofendida o degradada durante la asistencia a los servicios de salud, sintiendo que alguien ejerce chantaje en su contra o no muestra respeto por su opinión de tal manera que le haya molestado esta experiencia?

¿Alguna vez ha sentido que durante una visita normal a los servicios de salud, esta se ha convertido en una experiencia terrible o insultante sin saber el por qué?

¿Ha sentido que durante la atención en el servicio de salud alguien intencionalmente, le ha hecho daño físico, mental, se ha sobrepa-sado tocando su cuerpo en contra de su voluntad o la ha violado?

Tabla 2. Presencia de antecedentes de abuso emocional, físico, sexual y de los servicios de salud según el Cuestionario de Abuso de NorAQ en las Mujeres de la Comunidad de Tierra Baja (2013) 


\begin{tabular}{l} 
Factores de Riesgo \\
\hline Presencia de miembros de la familia con depresión, anteceden- \\
tes de suicidio o enfermedades mentales \\
Persona de la familia con depresión, antecedentes de suicidio o \\
enfermedades mentales
\end{tabular}

Ninguno

Esposo

Hermana

Hermano

$\mathrm{Hija}$

Cuñado

Presencia de violencia intrafamiliar en la familia de origen de la mujer

Presencia de violencia intrafamiliar en la familia de origen de su esposo

Dependencia económica de su esposo

Ingresos económicos insuficientes para satisfacer las necesidades básicas

Sobrecarga de roles como mujer

Desinterés del esposo para trabajar

Falta de recreación en la familia

Consumo de alcohol en familia

Persona que consumen alcohol en el interior del hogar

Ninguno

Esposo

Hijo

Padre

Hermano

Usted

Ambos padres

Gasto del salario en alcohol por parte del esposo

Rol del hombre

Formador de Hijos

Proveedor Económico

Formador de hijos y proveedor económico

Ninguno

Rol de la mujer

Formador de Hijos

Proveedor Económico

Formador de hijos y proveedor económico

\begin{tabular}{ccc}
$\begin{array}{c}\text { Frecuencia } \\
(\mathbf{N}=\mathbf{2 2 0})\end{array}$ & $\begin{array}{c}\text { Porcentaje } \\
(\%)\end{array}$ & IC $95 \%$ \\
\hline 7 & 3,2 & $0,8-5,5$
\end{tabular}

$\begin{array}{ccc}213 & 96,8 & 94,4-99,0 \\ 3 & 1,4 & 0,2-7,0 \\ 1 & 0,4 & 0,4-1,3 \\ 1 & 0,4 & 0,4-1,3 \\ 1 & 0,4 & 0,4-1,3 \\ 1 & 0,4 & 0,4-1,3 \\ 28 & 12,7 & 8,2-17,0 \\ 27 & 12,3 & 7,9-16,6 \\ 131 & 59,5 & 53,0-66,0 \\ 83 & 37,7 & 31,3-44,1 \\ 99 & 45,0 & 38,4-51,6 \\ 5 & 2,3 & 0,2-4,2 \\ 130 & 59,1 & 52,6-65,6 \\ 23 & 10,4 & 6,3-14,5\end{array}$

198

90,0

$86,0-93,9$

13

5,9

2,7-9,0

3

1,4

0,44-1,3

1

0,4

0,18-2,9

3

1,4

0,44-1,3

1

0,4

0,44-1,3

1

0,4

0,44-1,3

9

4,1

1,4-6,7

$\begin{array}{ccc}15 & 6,8 & 3,4-10 \\ 100 & 45,4 & 38,8-52,0 \\ 95 & 43,2 & 36,6-49,7 \\ 10 & 4,5 & 1,7-7,3\end{array}$

133

60,4

53,9-66,9

4

1,82

0,03-3,5

83

37,3

31,3-44,1

Tabla 3. Factores de Riesgo para la presencia de violencia intrafamiliar en las familias de las mujeres de la Comunidad de Tierra Baja (2013)

REV CLÍN MED FAM 2015; 8(1): 19-30 


\begin{tabular}{|c|c|c|c|}
\hline Variables & $\begin{array}{c}\text { Frecuencia } \\
(\mathrm{N}=220)\end{array}$ & $\begin{array}{l}\text { Porcen- } \\
\text { taje (\%) }\end{array}$ & IC $95 \%$ \\
\hline \multicolumn{4}{|l|}{ Conocimientos sobre violencia } \\
\hline Mujeres educadas sobre violencia contra la mujer & 54 & 25,4 & $19,6-31,2$ \\
\hline \multicolumn{4}{|l|}{ Acciones consideradas como violencia } \\
\hline Hablar en voz alta y con firmeza & 3 & 1,4 & $0,1-2,9$ \\
\hline Insultos, burlas, ridiculizar & 2 & 0,9 & $0,3-2,1$ \\
\hline Jalones, empujones & 3 & 1,4 & $0,1-2,9$ \\
\hline Golpes, palizas & 2 & 0,9 & $0,3-2,1$ \\
\hline Todas las anteriores & 210 & 95,4 & $92,6-98,2$ \\
\hline \multicolumn{4}{|l|}{ Principal agresor en el interior de un hogar } \\
\hline Hombre & 214 & 97,3 & $95,1-99,4$ \\
\hline Mujer & 6 & 2,7 & $0,5-4,8$ \\
\hline \multicolumn{4}{|l|}{ Factores considerados como de riesgo } \\
\hline Ausencia de diálogo & 43 & 19,5 & $14,2-24,8$ \\
\hline Educación de la persona & 62 & 28,2 & $22,1-34,1$ \\
\hline Alcohol, Drogas & 51 & 23,2 & $17,5-28,8$ \\
\hline Factores económicos & 18 & 8,2 & $4,5-11,8$ \\
\hline Falta de respeto & 35 & 15,9 & $11,0-20,7$ \\
\hline Estrés & 10 & 4,5 & $1,7-7,3$ \\
\hline Celos & 15 & 6,8 & $3,4-10,1$ \\
\hline \multicolumn{4}{|l|}{ Instituciones que manejan la violencia contra la mujer } \\
\hline Instituto Colombiano de Bienestar Familiar & 100 & 45,4 & $38,8-52$ \\
\hline Casa de Justicia & 14 & 6,4 & $3,1-9,6$ \\
\hline Puesto de policía cerca de su comunidad (Boquilla) & 14 & 6,4 & $3,1-9,6$ \\
\hline Comisaría de familia & 54 & 24,5 & $18,8-30,2$ \\
\hline Policía / Fiscalía & 51 & 23,2 & $17,5-28,9$ \\
\hline No sabe que institución & 7 & 3,2 & $0,8-5,5$ \\
\hline \multicolumn{4}{|l|}{ Actitudes frente a la violencia contra la mujer } \\
\hline \multicolumn{4}{|l|}{ Actitud frente un caso de violencia } \\
\hline No sabe manejar estas situaciones & 95 & 43,2 & $36,6-49,7$ \\
\hline Se queda callada & 31 & 14,1 & $9,4-18,7$ \\
\hline Toma una actitud sumisa y pasiva & 8 & 3,6 & $1,1-6,1$ \\
\hline Ninguna de las anteriores & 86 & 39,1 & $32,6-45,5$ \\
\hline \multicolumn{4}{|l|}{ Tipo de ayuda que buscaría } \\
\hline Consejo de familia & 169 & 76,8 & $71,2-82,4$ \\
\hline Consejo de amigos & 17 & 7,7 & $4,1-11,2$ \\
\hline Consejo de vecinos & 3 & 1,2 & $0,1-2,9$ \\
\hline Policía & 31 & 14,1 & $9,4-18,7$ \\
\hline
\end{tabular}

Tabla 4. Conocimientos, Actitudes y Prácticas de las Mujeres de la Comunidad de Tierra Baja sobre Violencia Intrafamiliar (2013) 


\begin{tabular}{|c|c|c|c|}
\hline Variables & $\begin{array}{c}\text { Frecuencia } \\
(\mathrm{N}=220)\end{array}$ & $\begin{array}{l}\text { Porcen- } \\
\text { taje (\%) }\end{array}$ & IC $95 \%$ \\
\hline Mujeres que denunciarían los casos de violencia & 219 & 99,5 & $98,6-100$ \\
\hline \multicolumn{4}{|l|}{ Prácticas frente a casos de violencia } \\
\hline \multicolumn{4}{|l|}{ Comportamiento frente a casos de violencia } \\
\hline Agredió a la persona que maltrataba & 26 & 11,8 & $7,5-16,1$ \\
\hline Se marchó de la casa & 9 & 4,1 & $1,4-6,7$ \\
\hline Se lo comunicó a un amigo o familiar & 88 & 40,0 & $33,5-46,5$ \\
\hline Denunció & 15 & 6,8 & $3,4-10,1$ \\
\hline Ideas o actos suicidas & 2 & 0,9 & $0,3-2,1$ \\
\hline Infidelidad & 2 & 0,9 & $0,3-2,1$ \\
\hline No ha visto ningún caso & 78 & 35,4 & $29,1-41,8$ \\
\hline \multicolumn{4}{|l|}{ Última causa de violencia en su hogar } \\
\hline Celos & 64 & 29,1 & $23,1-35,1$ \\
\hline Responsabilidad con los niños & 24 & 10,9 & $6,7-15,0$ \\
\hline Dinero & 20 & 9,1 & $5,2-12,9$ \\
\hline Efectos de alcohol o droga & 18 & 8,2 & $4,5-11,8$ \\
\hline Ninguna de las anteriores & 94 & 42,7 & $36,1-49,3$ \\
\hline Mujeres que involucran a los hijos en casos de violencia & 10 & 4,5 & $1,7-7,3$ \\
\hline Mujeres que asisten al psicólogo por sufrir de violencia & 4 & 1,8 & $0,03-3,5$ \\
\hline \multicolumn{4}{|l|}{ Razones por las que no denuncia } \\
\hline Miedo & 146 & 66,4 & $60,0-72,6$ \\
\hline Desconocimiento de la ley & 4 & 1,8 & $0,03-3,5$ \\
\hline Evitar problemas & 69 & 31,4 & $25,1-37,5$ \\
\hline Otra razón (confidencial) & 1 & 0,4 & $0,4-1,3$ \\
\hline
\end{tabular}

Tabla 4 (continuación). Conocimientos, Actitudes y Prácticas de las Mujeres de la Comunidad de Tierra Baja sobre Violencia Intrafamiliar (2013)

la familia de origen, baja escolaridad y consumo de alcohol; por otro lado se encontró ausencia de recreación, dependencia económica de las mujeres y disfuncionalidad familiar.

En relación a los conocimientos que presentan las mujeres con respecto al tema, la gran mayoría ha recibido información sobre el manejo de la violencia, y con respecto a las actitudes ante este comportamiento, declaran que buscarían ayuda de un familiar y denunciarían los casos de violencia. En cuanto a las prácticas realizadas frente a estos casos de violencia, se aprecia que lo primero que hacen es informar a un amigo cercano o familiar, pero no denuncian los casos ante las autoridades legales por miedo, y reportan los celos como la principal causa de los actos violentos (tabla 4).
Dentro de los mitos encontrados, la gran mayoría consideran que las mujeres son permisivas hacia los actos violentos.

Por otro lado, el hecho de estar casado/a, los ingresos familiares menores a un salario mínimo, la sobrecarga laboral de las mujeres, el consumo de alcohol del esposo, la falta de recreación y la disfuncionalidad familiar se mostraron asociados con la presencia de violencia intrafamiliar en el análisis univariante (tabla 5); pero al realizar el análisis multivariable, el modelo que mejor explica la presencia de violencia hacia la mujer incluye los siguientes factores: estado civil, falta de recreación de la familia y consumo de alcohol $\left(\chi^{2}: 22,26 ; p=0,0001\right)$ (tabla 5). 


\begin{tabular}{|c|c|c|c|c|c|c|c|}
\hline \multirow{2}{*}{$\begin{array}{l}\text { Factores relacionadas con violencia intrafami- } \\
\text { liar (VIF) }\end{array}$} & \multicolumn{2}{|c|}{$\begin{array}{l}\text { Presencia de } \\
\text { VIF }\end{array}$} & \multicolumn{2}{|c|}{$\begin{array}{l}\text { Ausencia de } \\
\text { VIF }\end{array}$} & \multirow[t]{2}{*}{ OR } & \multirow[t]{2}{*}{ IC $95 \%$} & \multirow[t]{2}{*}{$\mathbf{p}$} \\
\hline & $\mathbf{n}$ & $\%$ & $\mathbf{n}$ & $\%$ & & & \\
\hline \multicolumn{8}{|l|}{ Estado civil } \\
\hline Mujeres solteras & 11 & 35,5 & 20 & 64,5 & $2,5^{*}$ & $1,1-5,7$ & 0,02 \\
\hline Mujeres casadas o en unión libre (ref.) & 34 & 18 & 155 & 82 & 1,0 & - & \\
\hline \multicolumn{8}{|l|}{ Ingresos económicos de las Familias } \\
\hline Menores a un salario mínimo & 34 & 24,8 & 103 & 75,2 & 1,2 & $0,5-2,5$ & 0,5 \\
\hline Mayores a un salario mínimo (ref.) & 11 & 13,2 & 72 & 86,7 & & & \\
\hline \multicolumn{8}{|l|}{ Sobrecarga laboral en el hogar } \\
\hline Presencia & 29 & 29,2 & 70 & 70,1 & 2,7 & $1,3-5,3$ & 0,004 \\
\hline Ausencia (ref.) & 16 & 13,2 & 105 & 86,7 & 1,0 & - & \\
\hline \multicolumn{8}{|l|}{ Desinterés por parte del esposo en trabajar } \\
\hline Presencia & 3 & 60 & 2 & 40 & 6,1 & $1-3,8$ & 0,05 \\
\hline Ausencia (ref.) & 42 & 19,5 & 173 & 80,4 & 1,0 & - & \\
\hline \multicolumn{8}{|l|}{$\begin{array}{l}\text { Gasto del sueldo en alcohol por parte del } \\
\text { esposo }\end{array}$} \\
\hline $\mathrm{Si}$ & 6 & 66,7 & 3 & 33,3 & 8,8 & $2,1-3,6$ & 0,003 \\
\hline No (ref.) & 39 & 18,4 & 172 & 81,5 & 1,0 & - & \\
\hline \multicolumn{8}{|l|}{ Falta de recreación familiar } \\
\hline $\mathrm{Si}$ & 36 & 27,7 & 94 & 72,3 & $3,4^{*}$ & $1,5-7-5$ & 0,002 \\
\hline No (ref.) & 9 & 10 & 81 & 90 & 1,0 & - & \\
\hline \multicolumn{8}{|l|}{$\begin{array}{l}\text { Consumo de alcohol por algún miembro de la } \\
\text { familia }\end{array}$} \\
\hline $\mathrm{Si}$ & 10 & 43,4 & 13 & 56,5 & $3,5^{\star}$ & $1,4-8,7$ & 0,006 \\
\hline No (ref.) & 35 & 17,8 & 162 & 82,2 & 1,0 & - & \\
\hline \multicolumn{8}{|l|}{ Disfuncionalidad Familiar } \\
\hline $\mathrm{Si}$ & 36 & 26,47 & 100 & 73,53 & 3 & 1,3-6,6 & 0,006 \\
\hline No & 9 & 10,71 & 75 & 89,29 & & & \\
\hline
\end{tabular}

*Valores del modelo multivariado: $X^{2}: 22,2 ; p=0,001$.

Tabla 5. Asociación entre Violencia Intrafamiliar y los factores relacionados con su presencia en las mujeres de la Comunidad de Tierra Baja (2013)

\section{DISCUSIÓN}

Este estudio es el primero en su género que se realiza en poblaciones afro descendientes de la ciudad de Cartagena. Su diseño fue concebido de acuerdo a la actual necesidad del país para contar con líneas de base sólidas que permitan tomar decisiones en cuanto a la prevalencia del maltrato hacia la mujer y sus posibles factores de riesgo. Aunque con el presente diseño transversal no es posible establecer causalidad con algunos facto- res, por la falta de temporalidad, especialmente en aquellos que cambian con el tiempo, los hallazgos obtenidos se pueden considerar una aproximación a la asociación y permiten explorar tendencias en este aspecto. Otra limitación del presente estudio se debe posiblemente al subregistro que se presenta con el uso de estos instrumentos de papel, los cuales, por la naturaleza íntima de la información, ya sea por vergüenza o por temor, pueden incluir algunos sesgos, que son imposibles de controlar en el análisis. A pesar de esto, aspectos de 
fortaleza como el tamaño de la muestra y la estandarización y validación previa de los cuestionarios permiten afianzar la validez de los hallazgos y lograr importantes interpretaciones a la luz de las exploraciones realizadas.

Según los resultados del presente estudio, las mujeres, principales cuidadoras en la comunidad de Tierra Baja, experimentan con frecuencia episodios de violencia, confirmando este fenómeno social en la ciudad de Cartagena, aunque con cifras un poco más bajas que las reportadas en Colombia a nivel nacional. ${ }^{6}$ Este fenómeno estuvo asociado principalmente a la falta de recreación de la familia y al consumo de alcohol por parte de la pareja, datos similares a los encontrados por Tuesca y Borda, en 2003, en Barranquilla, Colombia. ${ }^{19}$ La convivencia familiar genera muchas tensiones en la pareja, ubicando al hogar como el escenario donde la mujer puede verse más expuesta a situaciones de violencia. Muchas de las mujeres de Tierra Baja son amas de casa exclusivamente, dedicadas al cuidado de los niños, lo que junto a la dependencia económica y a la falta de recreación en la familia, genera un ambiente rutinario, tenso, cargado de estrés acumulativo, que predispone a la pareja a situaciones de conflictos y a los niños a sufrir maltrato infantil. Un estudio cualitativo realizado en México por Miranda ${ }^{20}$, y otros estudios publicados por Martínez $z^{21}$ en Cartagena y Gil22 en Cuba, reportan que las mujeres amas de casa sufren mayor tipo de violencia que aquellas que además trabajan fuera del hogar, aunque resulta contrario a las encuestas nacionales de Nicaragua, donde no se halló asociación significativa entre la ocupación de las mujeres y la violencia. ${ }^{23}$ La vida de la mujer cuidadora se condiciona por su papel. El $68 \%$ de las cuidadoras percibe que su trabajo en la casa afecta de manera importante la salud, su vida social y personal, informando que a menudo se encuentran cansadas, deprimidas y que se han visto obligadas a disminuir su tiempo de descanso. ${ }^{24-26}$

La población de Tierra Baja se considera una comunidad vulnerable, con habitantes afrodescendientes, que reflejan una ausencia de organización comunitaria, acompañada de una escasa infraestructura física para desarrollar actividades deportivas o de recreo, lo que conlleva a un uso no óptimo del tiempo libre, predispone al consumo de sustancias psicoactivas, fundamentalmente alcohol, y a la aparición de actos y comportamientos delictivos. ${ }^{7}$ Estos comportamientos propios de algunos cartageneros durante los fines de semana, junto al calor de la ciudad y la extroversión de sus habitan- tes, han provocado un bajo crecimiento personal en los habitantes de Tierra Baja, en especial en los hombres, desde edades tempranas, que ha propiciado en algunos hogares, ambientes de disputa, reclamos e insultos entre las parejas luego de los efectos del alcohol, según lo estipula el $\operatorname{COSED}^{7}$, comprometiendo también muchas veces la estabilidad emocional y física de los niños en el hogar.

El tipo de violencia más presentado fue la violencia psicológica o emocional y la causa principal reportada, fueron los celos, hallazgos similares a los resultados reportados por Gallardo y Sánchez en 2009, que señalan que una de cada diez familias ha dado evidencia de violencia entre sus miembros, específicamente la violencia psicológica. ${ }^{27}$ Por otra parte, se evidenció que la principal persona identificada como agresor fue el esposo, de forma similar al estudio realizado por Martínez en 2009. ${ }^{21}$

Según lo reportado por las mujeres del estudio, la gran mayoría de los hombres de esta comunidad presentan patrones de crianza no adecuados, basados en gritos e insultos en sus familias de origen, presentando dificultad en la comunicación y diálogo. Posteriormente, esta educación se perpetúa hasta sus descendientes y facilitaría la aceptación como normal de este comportamiento entre la comunidad. La violencia psicológica, al no dejar huellas visibles inmediatas, conlleva implicaciones más trascendentes, lo que permite que sea de más fácil aparición y permanencia. Pero debe tenerse en cuenta que, por lo general, precede a la violencia física y, por ende, debe ser intervenida a tiempo para evitar daños y secuelas más complejas. Destruir la autoestima de una persona sistemáticamente, mediante órdenes o respuesta utilizando tonos de voz fuertes y violentos, o a través de insultos, humillaciones, ofensas, desprecios, etc., también es una forma de violencia. ${ }^{28}$

A partir de todos los hechos violentos vividos en Colombia, es observada la solución de conflictos a través de la violencia. Dentro de las víctimas, por sus condiciones de vulnerabilidad, se encuentra la mujer; ésta, acompañada de factores de riesgo como la dependencia económica, la baja escolaridad y la poca protección por parte de las entidades del estado, se desvaloriza y adopta una posición "tolerante", con el ánimo de sobrellevar la dinámica familiar, recayendo el peso de la funcionalidad familiar sólo sobre sus hombros, no compartiendo esta responsabilidad con su pareja y facilitándose así la permanencia de la violencia en el hogar. Esto lo confirma la presencia de algunos mitos reportados en este estudio, que visibilizan la aceptación 
de la violencia como estilo de vida y como manera natural de conducir una relación de pareja.

Además, hay que tener en cuenta los antecedentes de violencia que han padecido los afrodescendientes en Cartagena de Indias, que cultivaron en estas comunidades sentimientos de baja autoestima, vulnerabilidad y aceptación del maltrato injustificado. ${ }^{28}$ Las mujeres afro descendientes, histórica y socialmente, han padecido la vulneración de sus derechos como personas dignas en distintos planos: a nivel personal, cultural, económico, laboral, sexual, reproductivo, y durante siglos han experimentado toda una transgresión e invisibilización de los principios de integridad, libertad y dignidad. ${ }^{28}$ Hoy, en el mundo contemporáneo, se une a esta lista la violencia de género aumentada, profundizada cada día más, por la incapacidad de muchos hombres de entender y aceptar que las mujeres no poseen dueños y que presentan los mismos derechos que ellos. ${ }^{21}$

Por otro lado, a pesar de contar las mujeres con el conocimiento sobre la identificación de actos de violencia, la mayoría desconocen cuáles son los mecanismos que les proporciona la ley para garantizar su protección en caso de ser víctimas de VIF, ya que muchas recurren al Instituto Colombiano de Bienestar Familiar, entidad que protege al menor y la familia, pero realmente la entidad de primera elección en Colombia corresponde a las Comisarías de Familia, por lo que se requiere mejorar la información al respecto. ${ }^{29}$ Lamentablemente, la concepción de muchos de los habitantes de Tierra Baja frente a esta problemática es indiferente, dada la ausencia de figuras colectivas comunitarias que muestren una posición de apoyo a esta problemática. Muchas mujeres no denuncian los casos de VIF, por miedo, coincidiendo con el estudio de Vásquez en 2008, en la ciudad de Barranquilla (Colombia), que concluye que este es el motivo para que no se diera a lugar las medidas necesarias para que las mujeres hicieran valer sus derechos, protección y seguridad en sus hogares. ${ }^{30}$

En Colombia, aún se debe trabajar en fortalecer la red social de apoyo de las mujeres frente a la violencia que las motive a denunciar y les brinde protección y cuidados posteriores a la denuncia, paralelo al trabajo psicosocial que se debe iniciar con ellas, en búsqueda de fortalecer su autoestima y reiniciar su proyecto de vida. La violencia es una realidad que, aunque presente en la vida cotidiana, tiende generalmente a ocultarse por muchas razones, entre ellas, la vergüenza, las amenazas de más actos violentos o de muerte, el miedo a perder la seguridad económica y la de los hijos, la resignación a ese destino, las creencias religiosas y la impunidad, entre otras.

En el presente estudio, la población encuestada considera que tras la presentación de estos actos, las víctimas en la mayoría de los casos son los niños, resultado muy similar a las investigaciones ya citadas de Vásquez, que reporta que los más afectados con este hecho son los menores de edad, agredidos ya sea por sus padres o por quien los cuida o por una tercera persona con la aquiescencia de un familiar. Los hogares de estas mujeres presentan disfuncionalidad familiar y la presencia de muchos factores de riesgo hacia la violencia; si no se logran eliminar o controlar, estará en riesgo el bienestar de los núcleos familiares. Si se pretende trabajar con el cuidado de la salud general de los niños y de sus madres y se es indiferente a su dinámica familiar, no se dispondrá de un ambiente adecuado que propicie hábitos y conductas saludables a los niños y se desaprovecharan todas las actividades y esfuerzos realizados en la creación y búsqueda de una cultura saludable.

Al conocer la actual situación que viven las mujeres, cuidadoras principales de los niños de la población de Tierra Baja, se hace necesario difundir los resultados entre los diferentes actores sociales competentes, con el fin de diseñar intervenciones enfocadas a trabajar aquellos factores de riesgo de violencia que puedan desequilibrar el hogar y ser implementadas simultáneamente a las actividades desarrolladas en torno al cuidado de la salud. Una recomendación puede ser la realización un instrumento que categorice anticipadamente el nivel de riesgo de violencia de una mujer, para su intervención oportuna. Se resalta la utilidad del cuestionario NorAQ en el estudio, al ofrecer una estandarización de los tipos de abuso a presentarse en las mujeres. Es importante concienciar a las mujeres sobre sus capacidades y limitaciones, como un ser capaz de promover cambios sociales importantes en la comunidad y como eje fundamental en el cuidado y desarrollo adecuado de la población infantil.

\section{BIBLIOGRAFÍA}

1. United Nations Economic and Social Council, Report of the Special Rapporteur on violence against women. Further promotion and encouragement of human rights and fundamental freedoms, including the question of the programme and methods of work of the commission alternative approaches and ways and means within the United Nations system for improving the effective enjoyment of human rights and fundamental freedoms. E/CN.4/1996/53, 
February 1996. Disponible en: http://www1.umn.edu/humanrts/commission/thematic52/53-wom.htm [consultado el 01-02-2015]

2. Ayala Salgado L y Hernández Moreno K. La violencia hacia la mujer. Antecedentes y aspectos teóricos. Contribuciones a las Ciencias Sociales, Mayo 2012. Disponible en: http://www.eumed.net/rev/cccss/20/ashm.html [consultado el 04-02-2015]

3. Hsieh, SW. Femicidio, un indicador de violencia social hacia la mujer. Med Leg Costa Rica. 2012;29(1):79-82. Disponible en: http://www.scielo.sa.cr/pdf/mlcr/v29n1/art9.pdf [consultado el 10-10-2014]

4. Blitchtein Winicki D, Reyes Solari E. Factores asociados a violencia física reciente de pareja hacia la mujer en el Perú, 2004-2007. Rev. perú. med. exp. salud pública. 2012; 29(1):35-43.

5. OMS. Estimaciones mundiales y regionales de la violencia contra la mujer. Prevalencia y efectos de la violencia conyugal y de la violencia sexual no conyugal en la salud. 2013. Disponible en: http://apps.who.int/iris/ bitstream/10665/85243/1/WHO_RHR_HRP_13.06_spa. pdf?ua $=1$ [consultado 06-02-2015]

6. Instituto Nacional de Medicina Legal y Ciencias Forenses. Homicidio de mujeres en los primeros seis meses de 2013. Recuperado de http://www.medicinalegal.gov.co/index. php/oficina-de-prensa/662-homicidios-de mujeres-en-losprimeros-seis-meses-de-2013. [consultado el 06-02-2015]

7. Centro de Observación y Seguimiento del Delito COSED. Diagnóstico General de la situación de violencia y criminalidad en Cartagena de Indias Primer Semestre de 2013. Disponible en: http://www.cosed.org/a/wp-content/ uploads/2013/08/Diagnostico-a-junio-2013-Web.pdf [consultado el 8-02-2015]

8. Klevens, Joanne. Violencia física contra la mujer en Santa Fe de Bogotá: prevalencia y factores asociados. Rev Panam Salud Pública. 2001; 9(2):78-83.

9. Marrero Laceria V. La familia como objeto de estudio en el proceso de salud-enfermedad de los trabajadores. Rev Cubana de Salud y Trabajo. 2004; 5(1):62-7.

10. Puyana A. Género y familia. Asociaciones necesarias. Observatorio de Asuntos de Género. La familia. Reflexiones, Cifras y Legislación sobre Violencia Intrafamilar. Bogotá: Consejería Presidencial para la Equidad de la Mujer; 2004.

11. Ramírez CC. La sagrada violencia intrafamiliar. Corporación Sisma Mujer. 2006. Disponible en: http://www.sismamujer.org/sites/default/files/publicaciones/La\%20sagrada\%20violencia\%20intrafamiliar.pdf. [consultado el 4 de abril de 2010].

12. Lafaurie M. La violencia intrafamiliar contra las mujeres en Bogotá: una mirada de género. Revista Colombiana de Enfermería. 2013; 8(8):98-111.

13. Devries KM, Kishor S, Johnson H, Stockl H, Bacchus LJ, Garcia-Moreno $\mathrm{C}$ et al. Intimate partner violence during pregnancy: analysis of prevalence data from 19 countries.
Reprod Health Matters. 2010;18(36):158-70.

14. Medina, M, Borges GG, Lara C. Prevalencia de sucesos violentos y de trastorno por estrés postraumático en la población mexicana. Salud Pública Mex. 2005;47:8-21.

15. Katarina, S. The NorVold Abuse Questionnaire (NorAQ) Validation of new measures of emotional, physical, and sexual abuse, and abuse in the health care system among women. Eur J Public Health. 2003,13(4):361-366.

16. Smilkstein G. The family APGAR: A proposal for a family function test and its uses by physicians. J Fam Pract. 1978; 6:12-31.

17. Greenland S. Modeling and variable selection in epidemiologic analysis. Am J Public Health. 1989;79:340-9.

18. Hosmer DW, Lemeshow S. Applied logistic regression. 2nd edition. New York: Jhon Wiley and Sons Inc; 2000.

19. Tuesca R., Borda M. Violencia física marital en BarranquiIla (Colombia): prevalencia y factores de riesgo. Gac Sanit (revista en Internet). 2003 Jul); 17(4): 302-308. Disponible en: http://scielo.isciii.es/scielo.php?script=sci_arttext\&pid $=$ S021391112003000400008\&lng=es. [consultado el 20-52014]

20. Miranda L, Hapering D, Limón F, Tuñón E. Características de la violencia doméstica y las respuestas de las mujeres en la comunidad rural del municipio de Margaritas. Salud Mental. 2006;21(6):19-26.

21. Martínez, A. Cartageneras, cifras y reflexiones. Observatorio de Derechos Sociales y Desarrollo. Anaqueles de Ciudad. Género y Derechos Humanos. 2009;1:28.

22. Gil O. Situación de la violencia doméstica en la mujer, en la población del policlínico "Julio Antonio Mella". Rev Cubana Med Gen Integr. 2011;27(4):486-494.

23. OPS. El maltrato de las mujeres en edad fecunda en $\mathrm{Ni}-$ caragua. Oficina regional para las Américas de la OMS. Washington, DC.; 2004. p. 1-2.

24. Torres Egea P, Ballesteros Pérez E, Sánchez Castillo PD, Gejo Bartolomé A. Programas, intervenciones y redes de apoyo a los cuidadores informales en salud. Nursing. 2008;26(6):56-61.

25. Vaquiro S, Stiepovich J. Cuidado informal, un reto asumido por la mujer. Cienc. Enferm. 2010;16(2):17-24.

26. García MM, Mateo I, Maroto G. El impacto de cuidar en la salud y en la calidad de vida de las mujeres. Gac Sanit. 2004;18(2):83-92.

27. Larrain S. Estudio de prevalencia de la violencia intrafamiliar y la situación de la mujer en Chile. Santiago de Chile: Ed. Publicaciones de Salud Mental, 2005. p.38.

28. Murphy CM. Treating perpetrators of adult domestic violence. Md Med J. 1996;43(10):87.

29. Guía de Atención de la Mujer Maltratada. Norma Técnica 412 del 2000. Ministerio de Salud. Ministerio de Salud Dirección General de Promoción y Prevención, Colombia.

30. Vásquez, M; Figueroa, M. Violencia intrafamiliar: efectividad de la Ley en el Barrio de las Flores de la ciudad de Barranquilla. Rev de Derecho Universidad del Norte. 2008;29:196. 